\title{
Complete absence of the inferior vena cava presenting as a paraspinous mass
}

\section{LEE B MILNER AND ROBERTO MARCHAN}

From the Department of Radiology, Georgetown University Hospital, Washington DC, USA

Dilatation of the azygos-hemiazygos venous system presenting as paravertebral masses may be seen in intrahepatic and extrahepatic portal vein obstruction, anomalous pulmonary venous return, occlusion of the superior and inferior vena cava, persistence of the left superior vena cava, hepatic vein obstruction, elevation of central venous pressure as in congestive heart failure, pericardial effusion, constrictive pericarditis, pregnancy, and isolated aneurysms of the azygos vein. ${ }^{1}$ Although prominence of the azygos vein is a wellknown finding with infrahepatic interruption of the inferior vena cava or persistence of the supracardinal vein, it has not been described to our knowledge in complete absence of the inferior vena cava.

\section{Case report}

The patient is a 32-year-old woman who was in excellent health until 1 August 1977, when she noted

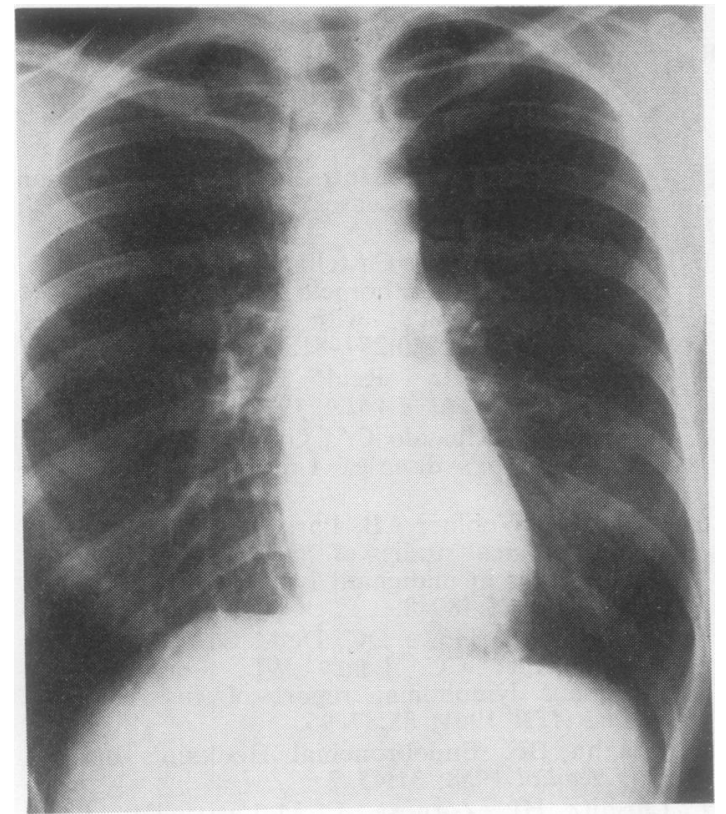

Fig $1 P A$ chest radiograph at the time of the patient's hospital admission. Note the prominent left paraspinous mass (arrow).

Address for reprint requests: Dr Lee B Milner, Department of Radiology, Georgetown University Hospital, 3800 Reservoir Road, NW Washington DC 20007, USA.

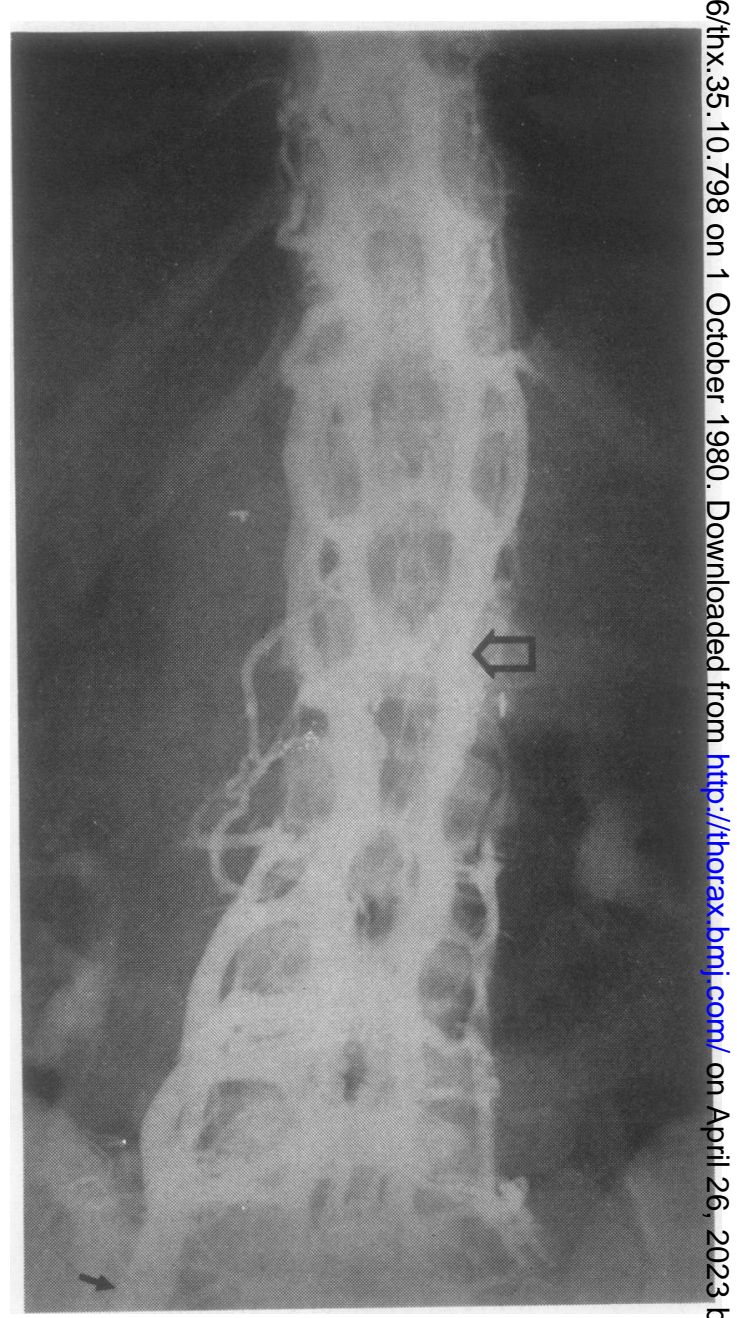

Fig 2 Selected film from the patient's inferior vena cavagram. The injection was made from the right iliac vein (small arrow). Contrast material directly entered the paravertebral plexus of Batson (large arrow).

right upper quadrant pain and fever. Investigatio revealed normal complete blood count, sedimentatich rate, liver function tests, uric acid, oral cholecyst ${ }_{-}=$ gram, barium meal, abdominal CT scan, intravenous pyelogram, lumbar puncture, and abdominal arterig- 


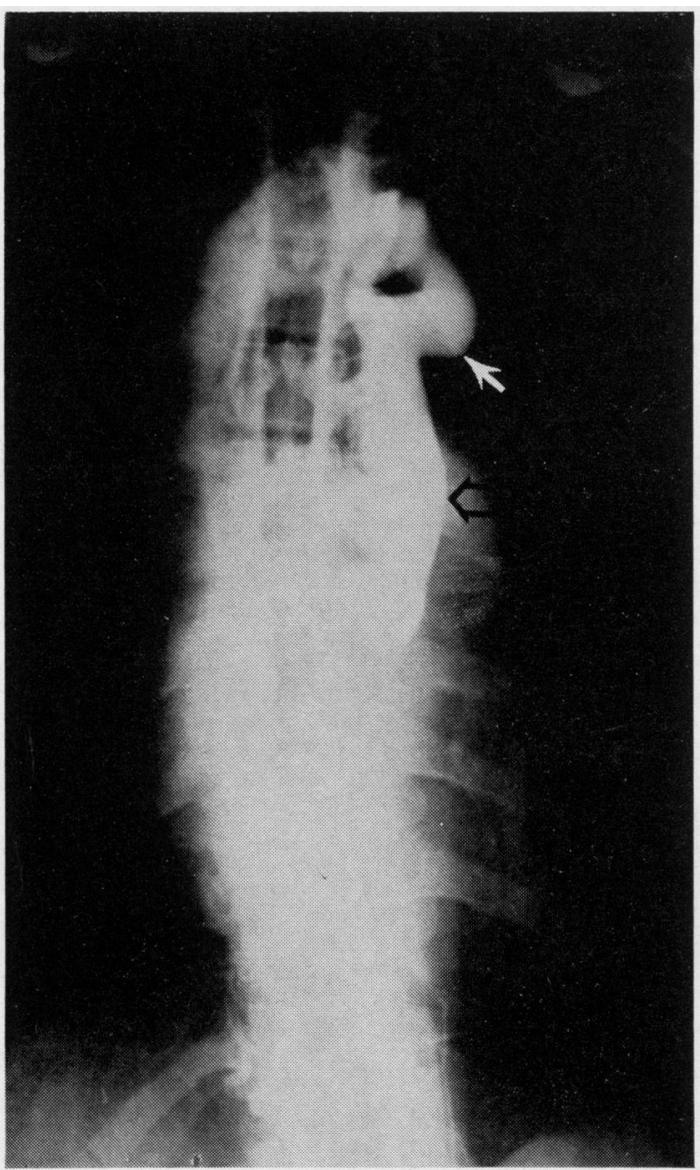

Fig 3 Anteroposterior view of the chest made during injection of the right iliac vein. The accessory

hemiazygos vein (large arrow) which corresponds to the paraspinous mass noted on the plain film of the chest empties into the left superior intercostal vein (small arrow).

gram. An hepatic sonogram demonstrated several cystic lesions. A liver biopsy was unsuccessful. The patient's pain occurred intermittently for the next two months and was associated with anorexia and an 11 pound weight loss. Because of these symptoms, the patient was admitted to Georgetown University Hospital on 15 September 1977. Of note in the patient's past history was a pelvic staphylococcal infection from suturing of the cervix to prevent miscarriage in 1973. The infection persisted despite therapy and led to the loss of the fetus and partial hysterectomy. A chest radiograph revealed a left paraspinous mass at T5-T8 (fig 1). The chest films of 1973 showed a similar appearance. The Gallium and CT scans were negative. Ultrasound again demonstrated multiple cystic lesions which appeared to be within the liver. Liver biopsy was negative. An exploratory laparotomy was performed on 3 October 1977. The liver was normal and free from cysts. The remainder of the exploration was also normal except for absence of an inferior vena cava. An extremely tortuous vein arose from the right renal vein and extended superiorly posterior to the liver.

A postoperative inferior vena cavagram was performed from the right femoral vein. Contrast material flowed from the right iliac vein directly into the paravertebral plexus of Batson (fig 2) and from there ascended into the azygos and hemiazygos systems. A large left paraspinous vein, probably the accessory hemiazygos vein filled, and emptied into the left superior intercostal vein to drain into the left in-

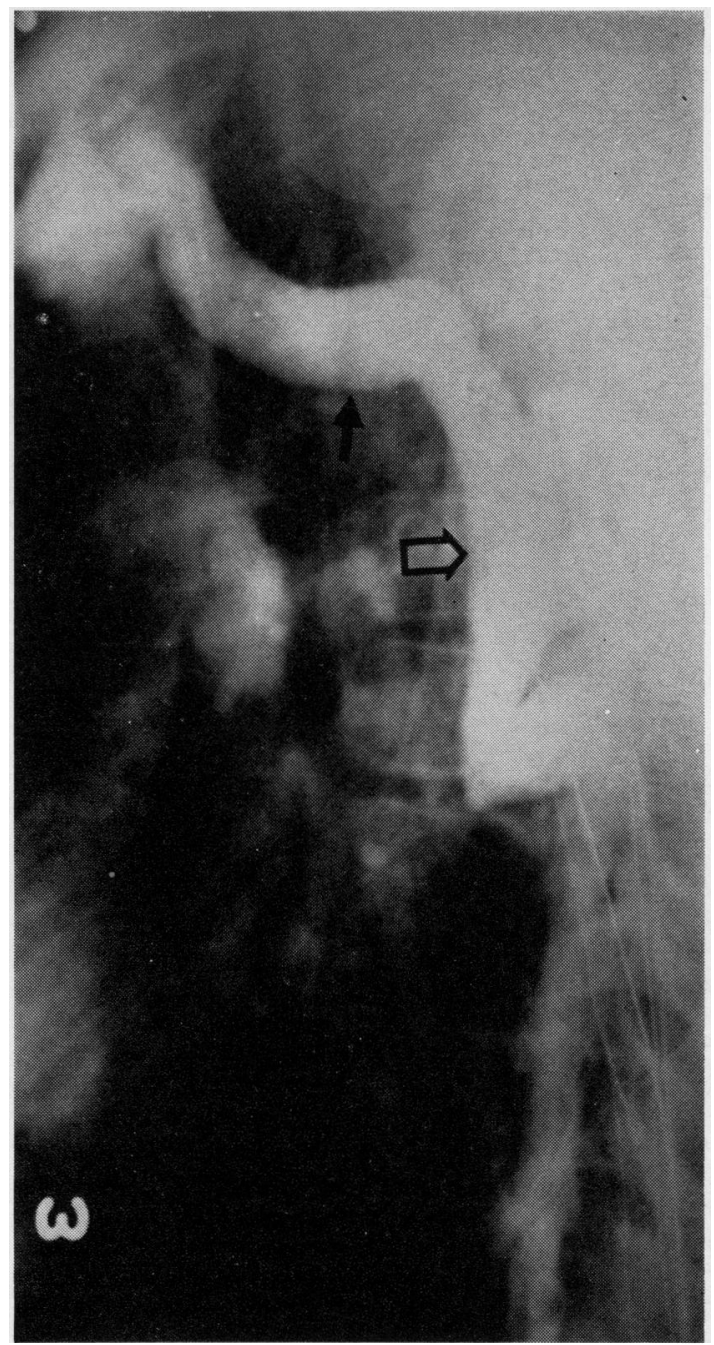

Fig 4 Lateral view of the chest made during right iliac vein injection. The accessory hemiazygos vein (large arrow) empties into the left superior intercostal vein (small arrow). 
nominate vein and superior vena cava (figs 3,4 ). The large accessory hemiazygos vein corresponded to the mass noted on the chest radiograph. There was no visualisation of the inferior vena cava. The patient remained afebrile with mild right upper quadrant pain of unknown aetiology.

\section{Discussion}

There is good surgical evidence that no left or right inferior vena cava was present. It might have been helpful to have performed a left femoral vein injection at the time of the inferior vena cavography. ${ }^{2}$ However, because of clinical difficulty, this was not possible. The tortuous branch of the right renal vein probably explains the defect seen on the sonogram at the level of the liver. ${ }^{3}$ It would be difficult to regard the absence of the inferior vena cava as congenital, since the embryological right hepatic vein, the right subcardinal vein, the right sub-supracardinal anastomosis, and the right supracardinal vein would have all had to regress. ${ }^{4}$ Although this remains a possibility, inflammatory obstruction of the inferior vena cava from the previous pelvic staphylococcal

\section{Correspondence}

Reproducibility of the tlow-volume loop

Sir,- In their letter in your June issue, Shaw and Fisher comment generously on our study of flow-volume loop reproducibility. ${ }^{1}$ They rightly draw attention to the effect of instrument error on within-subject reproducibility. However, it is virtually impossible to isolate instrumentbased variance with human subjects as it is tangled up with the subjects' own variability. These two components combine vectorially - that is, the variances are added together. If within-subject variance is, say $10 \%$ for $\mathrm{MEF}_{50}$ and $15 \%$ for $\mathrm{MEF}_{75}$, these values fall to $9.4 \%$ and $14.6 \%$ if the instrument variance (assumed to be $3.5 \%$ ) is removed. Thus instrument variance represents only a small part of total within-subject variation. Shaw and Fisher show that instrument error is markedly higher at low flow rates. Despite this, $\mathrm{MEF}_{75}$ appears rather more reliable than $\mathrm{MEF}_{50}$ in our analysis. This again suggests that machine error may not be a large factor.

The measurement of helium isoflow volume is critically dependent on reliability at extremely low rates, especially infection seems more likely. Thus with occlusion of the entire inferior vena cava, the blood flow from the legs returns to the heart via a markedly dilated hem 5 azygos system which is apparent on the chest radio graph as a paraspinous mass.

We thank Ernest $J$ Ferris MD for reviewing th $\vec{P}$ manuscript.

\section{References}

1 Castellino RA, Blank N, Adams DF Dilatef azygos and hemiazygos veins presenting in paravertebral intrathoracic masses. $N$ Engl $J$ Meळ 1968; 278:1087-91.

2 Abrams HL. The vertebral and azygos venou systems, and some variations in systemic venous return. Radiology 1957; 69:508-26.

3 Ferris EJ, Vittimberga J, Byrne JJ, Nabseth DC Shapiro JH. The inferior vena cava after ligatiog and plication. A study of collateral routes Radiology 1967; 89:1-10.

4 Chuang VP, Mena CE, Hoskins PA. Congenita anomalies of the inferior vena cava. Review of embryogenesis and presentation of a simplifie $\&$ classification. Br J Rad 1974; 47:206-13.

in subjects with airflow obstruction. By extrapolatin their table, it appears that instrument error is likely to be substantial at these extremely low flow rates. Thu machine error may play a sizable part in the very pogr reproducibility of helium isoflow volume in our stud although it is unlikely to be a substantial factor for othero flow-volume loop parameters.

J B MACDONAL
T J COLE
Ayrshire Central Hospital,
Irvine and
Dunn Nutritional Laborator
Cambridge

\section{Reference}

1 Macdonald JB, Cole TJ. The flow-volume loo reproducibility of air and helium-based tests normal subjects. Thorax 1980; 35:64-9. 